\title{
Adolescent control beliefs: Cross-cultural variations of primary and secondary orientations
}

Rachel Seginer, Gisela Trommsdorff \& Cecilia Essau 
This article reports of two studies addressing the meaning of primary and secondary control beliefs for transition to modernity and modern adolescents. Study 1 participants $(N=365)$ were Malaysian (transition to modernity), and German and North American (modern) adolescents. Study 2 participants $(N=757)$ were Israeli Druze (transition to modernity) and Israeli Jewish (modern) adolescents. The control beliefs scales employed in the two studies drew from the primary-secondary control beliefs conceptualisation (Rothbaum, Weisz, \& Snyder, 1982), shared a similar Likert-type item structure, but differed in operationalisation. Analyses tested two hypotheses: (1) the value mediation hypothesis postulated that transition to modernity adolescents will score higher on secondary control beliefs and modern adolescents will score higher on primary control beliefs; (2) the double transition hypothesis postulated that transition to modernity adolescents will score higher on both primary and secondary control beliefs. Results supported these hypotheses only partly. However, they did show clearly that transition to modernity adolescents endorsed secondary control

Requests for reprints should be sent to Rachel Seginer, School of Education, University of Haifa, Haifa, 31905 Israel, or to Gisela Tregelogy, of Haifa, Haifa, 31905 Israel, or to Gisela Trom

An earlier version of this paper was presented at the 11 Biennial Meeting of the International Society for the Study of Behavioral Development. Minneapolis, MN, 3-7 July 1991. Study 2 was supported by a grant to the first author from the University of Haifa, JewishArab Center.

The valuable comments of J.R. Weisz on an earlier version of this paper and the three anonymous reviewers are here gratefully acknowledged. Also, the useful help of Hoda Heir and Haggai Kupermintz in carrying out the Israeli study are appreciated. The authors also wish to thank J. Jamieson (Lakehead University, Canada), K. Hart (Hofstra University, USA), and K. Schneewind (Munich University, Germany) for their help with the data collection for Study 1. 
beliefs more strongly than did modern adolescents. The discussion focuses on possible explanations of inconsistent results. It also suggests that future research should address two issues brought to light: the adaptive value of primary and secondary control beliefs; and the explanatory value of different control types. Both should be studied in historical, developmental, and cultural contexts.

\section{INTRODUCTION}

There is a general agreement among laypersons and professional developmentalists that adolescence is a time of transition. However, a growing number of adolescents around the world have been experiencing their developmental transition in the context of yet another transition: The cultural transition from traditionalism to modernity. Attending to these double transition adolescents, the main purpose of the present analysis is to examine their control beliefs by comparing them to the control beliefs of modern adolescents experiencing the single transition of "getting into life" (Beloff, 1986).

A basic premise of this research has been that subjective sense of control is of vital importance to psychological well-being (Rotter, 1966; Seligman, 1975). Hence, individuals will try to obtain control in different life domains (Paulhus, 1983), and in a variety of ways, compatible with their lifestyles, cultural traditions, and value orientations. Whereas earlier behaviouroutcome contingency models allowed to operationalise multiple control only in terms of multiple life domains, the two-process model of primary and secondary control (Rothbaum, Weisz, \& Snyder, 1982) suggested yet another dimension pertaining to intended person-environment fit. According to the Rothbaum et al. conceptualisation, primary control ensues from the activist, self-centred approach of Western thinking by which control is understood in terms of "the individual's ability to change the environment to fit the self's needs" (Rothbaum et al., 1982, p. 8). Secondary control dwells on the assumption that the self-environment fit can be achieved not only by a powerful self changing the environment, but also by the self changing her- or himself to fit in with existing reality.

Thus, the essence of personal control is not limited to the power to change the environment to the person's needs, but rather it applies more broadly to the multiple means by which intended person-environment fit can be obtained. In fact, the primary and secondary processes share a common agent of change-the self, but differ in the target of change. In the primary process, the target of change is the environment and in the secondary process, it is the self. However, the two processes do not stand on equal footing. Rather, the Rothbaum et al. (1982) assumption has been that the basic form of control is that obtained from changing the environment to fit the person. Only under certain conditions, when primary control is judged to be unobtainable or inappropriate, it is substituted by secondary control, drawn from changing the self to fit reality (see also Heckhausen \& Schulz, 1991).

\section{Four Types of Control}

Drawing on earlier studies of attributions in the face of perceived uncontrollability, Rothbaum et al. (1982) have suggested four types of control. Although this typology was prompted by their formulation of secondary control, Rothbaum et al. could also demonstrate the pertinence of each of the four themes to primary control. Addressing secondary control processes, Predictive control applies to the person's ability to predict uncontrollably aversive outcomes, and thus avoid their ensuing negative consequences. Illusory control pertains to individuals' tendency to "align themselves with the force of chance so that they may share in the control exerted by that powerful force" (Rothbaum et al., 1982, p. 17). Vicarious control pertains to control by proxy. That is, "by aligning themselves with more powerful others, individuals can share in their victories and in their accomplishments-in short, in their control" (Rothbaum et al., 1982, p. 20). Interpretive control relates to the tendency to seek explanation and meaning in the face of uncontrollable situations. Frankl's (1963) analysis of the therapeutic gains of attributing meaning and explanation to calamity is an example of interpretive control.

As noted by Rothbaum et al. (1982), these four types are conceptually related. Predictive and illusory control have a common element of passivity and withdrawal reactions, illusory and vicarious control are commonly obtained by association with powerful agents, and interpretive control shares with all the other types of control the merits of explanation and meaning. Altogether, this typology elucidates the different but related meanings of secondary control, sharing in common the idea that individuals can gain a sense of control by actively accepting environmental supremacy.

At present there have been only few attempts to operationalise these types of control and empirically test their differential attributes, employment, and effectiveness (Essau, 1992; Essau \& Trommsdorff, 1991; Weisz 1986). These analyses suggested that the conceptual relationships pointed out by Rothbaum et al. (1982) call for careful operationalisation of the control types (see also Study 2), and that the four types covary with cultural background (see also Study 1 ).

\section{The Sociocultural Context}

Given that the cultural context is multifaceted, the present analysis will attend to three issues. First, primary, and secondary control orientations are instantiated in cultural lifestyles. Bearing on evidence from several 
domains of daily life experienced by Americans and Japanese, Weisz, Rothbaum, and Blackburn (1984) have analysed the contrasting contro beliefs reflected in areas like childrearing, socialisation, religious beliefs, work attitudes, and psychotherapy. Their analysis demonstrated that American lifestyle is guided by primary control beliefs, and Japanese lifestyle by secondary control beliefs.

Secondly, the differential prevalence of the primary and secondary control beliefs is associated with cultural subscriptions to individualisticcollectivistic value orientations (Trommsdorff, 1989). At the core of this distinction (Schwartz, 1990; Hui \& Triandis, 1986) is the self-society hierarchical relationships and goal priorities. Both the Schwartz and the Triandis conceptualisations contended that individualistic (or contractual, Schwartz, 1990) societies endorse self-direction and stimulation, and collectivistic (communal, Schwartz, 1990) societies endorse tradition and restrictive conformity. According to Schwartz (1990, p. 144), self-direction is "independent thought and action-choosing, creating, exploring". Stimulation is "excitement, novelty and challenge"; restrictive conformity is "restraint of actions, impulses and inclinations likely to harm others or violate social expectations"; tradition is "respect, commitment, and acceptance of the custom and ideas that traditional culture or religion impose".

The relationships between value orientations and control orientations have been examined in a cross-cultural socialisation study (Essau \& Trommsdorff, 1990; Trommsdorff, 1989) comparing German and Japanese parents and their adolescent children. Findings indicated that the expectations of German parents for their children reflected individualistic value preferences, and those of Japanese parents reflected collectivistic value preferences. Specifically, German parents expected their children to be independent, have conflicts with their parents, and learn through sanctions, and Japanese parents expected their children to be submissive, learn through imitation, and maintain harmonious relationships with their parents. These differential expectations were associated with adolescents' control beliefs. Children of the German parents scored higher on primary control beliefs and children of the Japanese parents scored higher on secondary control beliefs.

Thirdly is the human condition of transition (Felner, Farber, \& Primavera, 1983). The conceptualisation of transitions in terms of changes and reaction to changes (Felner et al., 1983) implies the experience of new realities, governed by rules yet to be discovered. In other words, transitions involve uncertainty, impaired mastery of the situation, and loss of predictablity, all of which ensue a growing need to regain personal control. Specifically, as situations involved in cultural transitions become less familiar and hence their consequences harder to predict, as young adoles- cents experiencing puberty become less cognisant of their body and less familiar with social reactions to these changes (Petersen, 1985), or as the older adolescents face new tasks involved in moving from transition to adulthood roles in the realm of education to adulthood roles in the realm of career and family (Marini, 1984), they enter new realities where old rules do not apply, and the ability to control outcomes is therefore deemed curtailed.

Assuming linear relationships among transitions, uncertainty, and the need for control, the double transition experienced by transition to modernity adolescence produces a higher level of uncertainty, and hence a higher need for personal control. Moreover, the traditional components of their culture, make secondary control beliefs more available to transition to modernity adolescents than to modern adolescents.

Addressing the sociocultural context of primary and secondary control beliefs among adolescents, this report examines two hypotheses. The value mediation hypothesis dwelt on the relationships among lifestyles, value orientations, and control orientations. It postulated that because transition to modernity societies are guided by collectivistic value orientations and modern societies are guided by individualistic value orientations, members of these societies differentially endorse secondary and primary control beliefs. Thus, relative to modern adolescents, transition to modernity adolescents will score higher on secondary control beliefs and lower on primary control beliefs.

The double transition hypothesis postulated that because transition to modernity adolescents undergo both developmental (adolescence) and cultural (transition to modernity) transitions they are in a greater need for personal control. Thus, transition to modernity adolescents will score higher on both secondary and primary beliefs. These hypotheses were tested in relation to two sets of data. Study 1 compared the control beliefs of Malaysian adolescents as an instance of transition to modernity, and Canadian and North American and German adolescents as two instances of modern society. Study 2 compared the control beliefs of Israeli Druze and Jewish adolescents as instances of transition to modernity and modern societies, respectively.

\section{STUDY 1}

\section{Method}

Subjects. Participating in this study were 365 undergraduate university students (143 males and 222 females). Their national composition consisted of 112 Canadians, 50 Americans, 111 Germans, and 92 Malaysians The mean age of the North American group was $19.94(\mathrm{sd}=3.01)$, of the 
German group 24.67 (sd = 5.00), and of the Malaysian group 21.74 (sd = 1.57). However, $89 \%$ of the entire sample were still single. The majority $(71 \%)$ of the Malaysian participants were Moslems. Among the modern adolescents, $57 \%$ were Catholics, and $27 \%$ were Protestants.

Malaysia is a multi-ethnic country, but Malaysian universities are attended mainly by Iban and Malay students. Hence, the Malaysian sample-recruited from one of the major universities of Malaysiaconsisted mostly of Iban and Malay students. The two ethnic groups commonly live in villages where family dwellings are in close proximity. Both groups are also strongly influenced by their religions. The Iban religion has three central elements: supernatural powers, human beings, and rice (padi), and its main dictum demands harmonious relations between the supernatural (including spirits) and humans. The Malays are devout Moslems. Their children cite Koran verses from an early age and gradually assume religious duties like daily prayers and the Ramadan fast. Malaysian children are brought up to live according to adat, that is, an allencompassing customary system of rules, beliefs, norms, and values governing the physical and the supernatural world. The adat guarantees harmony among community members and the supernatural powers, and its breach may harm the entire community.

Instrument. The Primary Secondary Control Questionnaire (PSCQ) consists of 38 Likert-type items ranging from 1 ("do not agree") to 4 ("strongly agree"). The questionnaire contains two subscales: primary control; and secondary control. Each subscale comprises of equal number of items pertaining to the four types of control: predictive, illusory, vicarious, and interpretive. Examples of items pertaining to the four types of primary control are: "Before I work in foreign countries I like to learn the official language there so that I don't have much difficulty doing my work" (Predictive); "I gamble a lot in order to be good at it" (Illusory); "I seek close alignment with powerful others in order to use their strength to influence other people or events" (Vicarious); "When I feel sick I like to know of its cause in order to prevent it from occurring again" (Interpretive). The Cronbach alpha of the primary scale is 0.81 and 0.67 for the transition to modernity and modern samples, respectively. Examples of items pertaining to the four types of secondary control are: "I feel more comfortable going somewhere if I know what to expect ahead of time", (Predictive); "It is no point fighting against bad luck; instead one has to be at peace with what fate brings into one's life" (Illusory); "I make much effort to be friends of some important and powerful people in order to enhance my feelings of pride" (Vicarious); "I interpret my hardship as a process of learning more about myself and other people" (Interpretive). The Cronbach alpha of the secondary control scale is 0.75 and 0.84 for the transition to modernity and modern samples.
The PSCQ was constructed in English. Translations followed Brislin, Lonner, and Thorndike (1973) guidelines for the construction of questionnaires for cross-cultural studies. Thus, translations into German, Iban, and Malay (Malaysian languages) were performed by one bilingual person, and back-translations by another bilingual person. Differences between the original and the back-translated versions were discussed by the two translators, and resolved by mutual agreement (Essau, 1992; Essau \& Trommsdorff, 1991).

Procedure. Questionnaires were group administered by class instructors during regular sessions. The study was identically introduced as addressing "perceived control in different countries", and potential respondents were advised of the voluntary nature of their participation and the confidential treatment of their responses. Completed questionnaires were returned to Germany where scoring, coding, and analysis took place.

\section{Results}

Table 1 lists the means and standard deviations of the four types of primary control and secondary control beliefs, as well as the means and standard deviations of the total primary and secondary control beliefs scores for the three national groups. Comparisons employing the unique sum of squares MANOVAs demonstrated relatively high nationality effects for both the total primary and secondary control beliefs $(F(4,686)=65.23, P<0.001$, $\varepsilon^{2}=0.48$ ). That is, nationality uniquely (i.e. with gender held constant) explained $48 \%$ of the variance of the total primary and secondary control beliefs. MANOVAs for the four types of primary and secondary control beliefs showed similar effect of nationality $(F(16,674)=25.93, P<0.001$, $\left.\varepsilon^{2}=0.62\right)$, thus uniquely explaining $62 \%$ of the variance. Subsequent univariate analyses showed significant nationality effect for both the total primary and secondary control beliefs and for each of the four types of the primary and secondary control beliefs. However, the effect of gender was not significant. Hence, the post hoc comparisons were performed only for the three nationality groups.

The Tukey post hoc procedure showed consistent cross-cultural differences in the secondary control beliefs $(P<0.05)$. As hypothesised transition to modernity (Malaysian) adolescents scored significantly higher than the two modern groups. However, the two modern groups also differed significantly on all four types of secondary control as well as on the total, so that German adolescents scored lowest.

The Tukey post hoc procedure showed that the cross-cultural pattern of the primary control orientations is more complex. Both the value mediation and the double transition hypotheses postulated that the Malaysian group (transition to modernity) would score consistently differently than 
TABLE 1

Means and Standard Deviations (in parenthesis) of the Malayasian (M) North American (NA) and German (G) Groups

\begin{tabular}{lccccc}
\hline & Malaysia & $\begin{array}{c}\text { North } \\
\text { America }\end{array}$ & Germany & Univariate & $\begin{array}{c}\text { Post hoc } \\
\text { Comparisons } \\
(P=92)\end{array}$ \\
& $(N=162)$ & $(N=111)$ & $F(2,343)$ & $(P<0.05)$ \\
\hline Primary control (total) & 45.98 & 46.38 & 42.65 & $11.30^{* * *}$ & $\mathrm{G}<\mathrm{M}, \mathrm{NA}$ \\
& $(6.57)$ & $(6.64)$ & $(4.55)$ & & \\
Predictive & 15.65 & 15.83 & 14.98 & $4.93^{* *}$ & $\mathrm{G}<\mathrm{NA}$ \\
Illusory & $(2.63)$ & $(2.57)$ & $(2.13)$ & & \\
& 5.30 & 6.27 & 4.92 & $17.90^{* * *}$ & $\mathrm{M}, \mathrm{G}<\mathrm{NA}$ \\
Vicarious & $(1.58)$ & $(2.16)$ & $(1.24)$ & & \\
& 12.16 & 9.73 & 6.84 & $87.26^{* * *}$ & $\mathrm{G}<\mathrm{NA}<\mathrm{M}$ \\
Interpretive & $(2.15)$ & $(3.10)$ & $(1.69)$ & & \\
Secondary control (total) & 12.87 & 14.53 & 15.90 & $22.50^{* * *}$ & $\mathrm{M}<\mathrm{NA}<\mathrm{G}$ \\
& $(3.21)$ & $(2.74)$ & $(2.41)$ & & \\
Predictive & 51.52 & 44.03 & 34.50 & $116.90^{* * *}$ & $\mathrm{G}<\mathrm{NA}<\mathrm{M}$ \\
& $(5.85)$ & $(7.59)$ & $(6.02)$ & & \\
Illusory & 9.28 & 8.85 & 7.83 & $16.87^{* * *}$ & $\mathrm{G}<\mathrm{NA}<\mathrm{M}$ \\
& $(0.96)$ & $(1.76)$ & $(1.83)$ & & \\
Vicarious & 11.47 & 9.47 & 6.61 & $77.97^{* * *}$ & $\mathrm{G}<\mathrm{NA}<\mathrm{M}$ \\
& $(2.43)$ & $(2.45)$ & $(1.28)$ & & \\
Interpretive & 15.41 & 13.26 & 9.47 & $71.89^{* * *}$ & $\mathrm{G}<\mathrm{NA}<\mathrm{M}$ \\
& $(2.15)$ & $(3.10)$ & $(1.69)$ & & \\
& 15.34 & 12.43 & 10.55 & $57.00^{* * *}$ & $\mathrm{G}<\mathrm{NA}<\mathrm{M}$ \\
& $(3.21)$ & $(2.74)$ & $(2.41)$ & & \\
\hline
\end{tabular}

${ }^{* *} P<0.01 ;{ }^{* * *} P<0.001$

the two modern groups: The value mediation hypothesis predicted that the Malaysian respondents would score lower, and the double transition hypothesis predicted that they would score higher than their modern counterparts. However, these predictions were not sustained.

The Malaysian, and the North American adolescents, scored higher than the German adolescents on the total primary score. The Malaysians also scored significantly higher than both the North American and the German adolescent groups on the vicarious primary control, but scored significantly lower than the two modern adolescent groups on the primary interpretive control. The most consistent pattern emerged for the German adolescents who expressed relatively low primary control beliefs, thus also refuting the value orientation prediction that modern adolescents score higher on primary control. The results indicated that the differential salience of authority figures and self were also reflected in the primary control orientations. Transition to modernity adolescents more frequently expressed their primary control orientations by relating to the benefits accrued from alignment with powerful agents (vicarious), and modern adolescents by addressing the meaning of the situation at hand (interpetive).

Overall, the secondary control scale gave more consistent results, demonstrating that transition to modernity adolescents more highly endorse secondary control orientation. The reasons for the inconsistent pattern of results for the primary control scale may bear on the operationalisation of both the primary and the secondary control beliefs in terms of the four types. As noted by Rothbaum et al. (1982) the four types of control were developed to explicate the secondary control concept, and were also applied to the conceptualisation of primary control. Thus, the applicability of this typology to primary control operationalisation should be further investigated.

\section{STUDY 2}

\section{Method}

Subjects. Participating in this study were 308 (122 boys, 186 girls) Israeli Jewish and 449 (233 boys, 216 girls) Israeli Druze adolescents. Both samples consisted of 9th-graders $(n=416)$ and 12th-graders $(n=341)$. The mean age of the Druze 9th-graders was $14.72(\mathrm{sd}=0.48)$ and of the 12 th graders $17.70(\mathrm{sd}=0.55)$. The mean age of the Jewish 9th-graders was 15.07 ( $\mathrm{sd}=0.43)$ and of the 12 th-graders $17.93(\mathrm{sd}=0.44)$. The Jewish sample was drawn from a large high school, containing both junior and the enior high school divisions and served the entire community of a medium sized town (population 80,000) in northwestern Israel. About a third of the parents $(30 \%$ of the mothers and $32 \%$ of the fathers) had at least some college education; about $60 \%$ of the mothers held a paying job. The Druze sample was drawn from three regional high schools in northwestern Israel, where the Israeli Druze community has been living for the last 200 years. Parents' formal education was considerably lower than that of Jewish parents. Less than $10 \%$ of fathers and less than $1 \%$ of mothers of Jewish parents. Less than $10 \%$ of fathers and less than $1 \%$ of mothers attained at least some college education. Mothers were not expected and not trained to work outside their household, and hence only very few mothers (less than $3 \%$ ) held a paying job. However, this demographic information should be understood in terms of the Druze historical and cultural background.

The unique sociocultural features of the Druze community have been described recently by several researchers (Ben Dor, 1979; Seginer \& Halabi, 1991; Soffer, 1984). According to these accounts, Israeli Druzes dwell in rural communities but the majority of their adult work force have been earning its living outside of farming. Their language, food, folklore, 
and social norms are similar to those of their Arab neighbours. Moreover, Moslem leaders have accepted the Druze faith as one branch of Islam (Firru, 1984). However, historical disputes between Druzes and Arabs led to an alliance between Israeli Druzes and Jews, and to the application of conscription (originally intended for Israeli Jewish males and females) to Druze males. To resolve contradicting demands for national allegiance, Druzes have either aligned themselves with the Israeli or Arab groups, or have adopted a multifaceted identity consisting hierarchically of Druze, Israeli, and Arab components (Alscheich, 1978).

Close contacts with the Israeli Jewish society and deep devotion to their religion and religious leaders have set the Druze community into a process of transition to modernity. Their lives represent a blend of rural residence, modern households, growing appreciation of higher education and professional careers, and strong power of religious tradition and community and family authority. To date, the Israeli Druze community pledges allegiance to both Jewish Israel and Arab culture, and seeks ways to incorporate some aspects of modernity into Druze traditionalism.

Instrument. The Primary Secondary Control Scale (PSCS) employed in this study was developed in Israel. Like the PSCQ developed in Germany (Study 1), it draws on the conceptualisation of primary and secondary control as two forms of internal beliefs, differing in the target of the behaviour-contingent outcomes (Rothbaum et al., 1982; Weisz et al., 1984). Thus, the PSCS consists of a primary control and secondary control subscales. Each subscale contains 13 Likert-type items ranging from 1 ("strongly disagree") to 6 ("strongly agree").

Examples of primary control items are: "If I am not accepted in my preferred educational programme I will apply again and struggle to be accepted the following year"; "I think my success depends on what I do and how hard I work"; "I believe I am capable of contributing to society in my future work". Examples of secondary control items are: "When I choose my future occupation I will consider my parents' opinion because in important matters parents have the final say" (Interpretive); "I work hard on my homework so that the teacher/lecturer is happy with me" (Vicarious); "In the future I will have a prestigious occupation because such an occupation brings respect to the person" (Vicarious).

The construction of the PSCS went through several stages. Originally, secondary control was represented by two separate subscales: vicarious and interpretive, as the two main dimensions on which transition to modernity and modern adolescents were expected to differ. Items for these two subscales as well as for the primary control scale were written in Hebrew by Druze and Jewish researchers and translated into Arabic by a bilingual Druze student. Translation was checked by back-translation into
Hebrew. Item analysis indicated high intercorrelations between the two types of secondary control $(r=0.67$ and $0.73, P<0.001$ for the Druze and Jewish samples, respectively), thus suggesting that the present operationalisation did not distinguish between vicarious and interpretive secondary control, and the two subscales should be combined. In its final version, employed in the present study, the PSCS consists of two subscales: primary control (alphas $=0.71$ and 0.75 for the Druze and the Jewish samples, respectively) and secondary control (alphas $=0.78$ and 0.80 for the Druze and Jewish samples, respectively). These primary and secondary contro subscales are relatively unrelated $(r$ for the Druze sample $=-0.08$, ns; and for the Jewish sample $=0.11, P<0.05)$.

Altogether, the control beliefs scales employed in both studies drew from the primary-secondary control beliefs conceptualisation (Rothbaum et al., 1984; Weisz et al., 1984). However, independently constructed, the two scales differed in the operationalisation of the primary and secondary control beliefs. Study 1 addressed all four types (predictive, illusory, vicarious, and interpretive) for the construction of both the primary and the secondary control subscales. Study 2 employed a global approach for the construction of the primary control beliefs subscale, and did not address all four control types for the construction of the secondary control subscale. Moreover, working in different cross-cultural settings each study had to take special precautions of generating a set of items relevant for all cultures under examination. Overall, the secondary control beliefs subscales of the PSCQ (Study 1) and the PSCS (Study 2) differed in the omission of predictive control items from the PSCS and in focusing on two different aspects of interpretive control. Study 1 emphasised the hardship and pain aspect of secondary control, and Study 2 emphasised the general tendency to change one's views "as to better accept the world" (Rothbaum et al., 1982 , p. 26) and applied it specifically to adolescent-parent conflicts.

Nevertheless, the PSCQ (Study 1) and the PSCS (Study 2) had several similar items. To illustrate, both instruments contained primary control items referring to the necessary steps for school success: "Before I write an important examination, I like to work hard for it in order to avoid failure" (PSCQ, 18); “In my opinion, the best way to succeed and achieve high grades is to work hard in everything related to school" (PSCS, 2). In addition, three PSCQ $(2,10,15)$ and three PSCS items $(6,14,16)$ addressed the illusory themes of secondary control: "It is no point fighting against bad luck, instead one has to be at peace with what fate brings into one's life" (PSCQ, 2); "When I think about the future I think I will be successful because people who have luck are successful" (PSCS, 6).

Finally, both instruments also contained items pertaining to alignment with powerful people: "I make much effort to be friends of some important and powerful people in order to enhance my feelings of pride" (PSCQ, 24); 
"I like to be friends with some important and powerful people in order to make myself feel important" (PSCQ, 28); "I would like my spouse to come from a well-off family because this will enhance my social status" (PSCS, 3 ); "I will agree with those who think one should associate with strong and successful people” (PSCS, 23).

Procedure. Data were collected in one class session as part of a larger study concerning Israeli adolescents' future orientations. Questionnaires were written, presented, and responded to in the respondents' native langauge.

\section{Results}

The means and standard deviations of the primary and secondary control scales for Jewish and Druze 9th- and 12th-grade males and females are presented in Table 2. Comparisons using the unique sum of squares MANOVAs supported the double transition hypothesis postulating that transition to modernity adolescents will score higher on both primary and secondary control beliefs $\left(F(2,748)=86.14, P<0.001, \varepsilon^{2}=0.19\right)$. That is, ethnicity uniquely (i.e. with gender and age held constant) explained $19 \%$ of the variance of control beliefs. Subsequent univariate analysis suggested that Druze adolescents endorse more highly both primary $(F(1,749)=$ 166.00, $P<0.001)$ and secondary control beliefs $(F(1,749)=8.29, P<$ $0.01)$.

The interpretation of these findings in terms of response bias on part of the Druze adolescents is unlikely for three reasons: (1) as noted above, the correlation between the primary and secondary control beliefs for the Druze sample is very low $(r=-0.08)$ and nonsignificant; (2) Druze and Jewish adolescents share a similar tendency to score higher on primary control than on secondary control beliefs, and their means have similar standard deviations; and (3) the MANOVA also showed unique gender $\left(F(2,748)=11.16, P<0.001, \varepsilon^{2}=0.03\right)$ and age $(F(2,748)=8.93, P<$ $\left.0.001, \varepsilon^{2}=0.02\right)$ effects. Subsequent univariate analyses showed that females of both ethnic groups hold stronger primary control beliefs $(F(1,749=14.32, P<0.001)$, and males $(F(1,749)=7.49, P<0.01)$ and younger adolescents $(F(1,749)=16.19, P<0.001)$ of both ethnic groups hold stronger secondary control beliefs. The MANOVA also yielded an ethnicity $\times$ gender $\times$ age interaction effect $\left(F(2,748)=3.12, P<0.05, \varepsilon^{2}\right.$ $<0.01)$ indicating that older (12th-graders) Druze females hold stronger primary control beliefs $(F(1,749)=4.13, P<0.05)$. 


\section{DISCUSSION}

Analyses carried out in Studies 1 and 2 showed that cultural patterns held more clearly for secondary than for primary control beliefs, and that transition to modernity respondents expressed stronger secondary control beliefs than did their modern counterparts. The consistency of these results across the two studies is especially notable, considering that the two studies differed in the national groups representing transition to modernity and modern cultural settings, age of respondents, and instruments. This clear cross-cultural pattern was not borne out for the primary control beliefs.

Study 1 -comparing the control beliefs of Malaysian, North American, and German university students - showed that the primary control beliefs of the Malaysian students were stronger than those of their German counterparts (exception is the primary-vicarious subscale) and inconsistently different from the North American students. Study 2-comparing Israeli Druze and Jewish adolescents-showed that Druze adolescents expressed stronger primary control beliefs. Thus, the results of Study 2 supported the double transition hypothesis, and the results of Study 1 did not support the value mediation hypothesis.

Explanation of the primary control endorsement of transition to modernity and modern adolescents may take several directions, mainly related to the operationlisation of primary control and to respondents' personal characteristics. As noted earlier, the operationalisation of primary control in terms of the four types of control drew on the Rothbaum et al. (1982) suggestion that the typology-originally developed to explicate secondary control-may be applied also to primary control. However, the inconsistent differences among the three cultural groups' responses to the primary control scale, relative to their clear endorsement of secondary control indicates that the operationalisation of primary control beliefs, in terms of the four-fold typology, may raise problems of nonequivalence, not applying to the operationalisation of secondary control beliefs.

The personal characteristic to be considered is respondents' age. All except for one group of respondents, were adolescents; the Israeli Druze and Jewish respondents were in middle adolescence; and the Malaysian and the North American respondents were entering late adolescence. However, the Germans, according to their age, were entering young adulthood. It is possible that their postponed entrance into adulthood, indicated by their enrolment in an undergraduate programme and low ratio of being married, has led the German students to resort to a profile of low primary and low secondary control beliefs. At the opposite end are the Israeli Druze adolescents. Their response profile is discussed in a later section. At this point suffice it to say that Druze adolescents, and especially the 12th-graders, meet their many transitional tasks by endorsing both primary and secondary control beliefs.

In addition, sociocultural differences between the two samples representing transition to modernity, and among the three samples representing modern societies, should be noted. Of special significance is the position of the Israeli Druze as a minority among minorities (Ben Dor, 1979), keeping close contacts with the Israeli Jewish community and establishment, whilst maintaining a separate culture. This dual relationship of both instrumental dependence and cultural independence may also affect their tendency for higher endorsement of primary control beliefs

The conclusion that transition to modernity adolescents of different age groups and nationalities more strongly endorse secondary control beliefs bears on the personal adjustment functions of secondary control beliefs. At present, psychologists tend to agree on the primary control nature of control. That is, control is understood in terms of gaining desirable outcomes and avoiding undesirable outcomes by altering existing conditions. Secondary control serves only as a temporary replacement in situations where primary control cannot be exercised.

Specifically, one approach (Weisz, 1983, 1986) suggests that when existing realities are unalterable and primary control beliefs inadequate, secondary control beliefs become functional to personal adjustment. Moreover, the results of a developmental study (Band \& Weisz, 1988) demonstrated that secondary control beliefs are age-related and become more accessible as children enter early adolescence. These results may suggest that the employment of the culturally nonprevailing control beliefs (i.e. secondary control beliefs) is learned and their development is associated with social cognitive development in early adolescence. In a similar vein, Heckhausen and Schulz (1991), contended that secondary control strategies serve psychological well-being by supporting the selectiveness of primary control and buffering the ill effects of primary control failures.

Our findings, by which transition to modernity adolescents of different nationalities and age score higher than modern adolescents on secondary control beliefs, and to some extent also score higher on primary control beliefs, suggest a different approach. This approach contends that as the need arises, individuals can expand their control beliefs repertoire by orienting themselves to setting appropriate beliefs. Cultural differences concerning the self, especially as these are reflected in self-other relationships (Markus \& Kitayama, 1991) suggest that secondary control beliefs have more influence, and thus may be more readily employed, in transition to modernity societies than in modern societies. Thus, transition to modernity adolescents expand their repertoire of available control and respond to uncertainties arising in both their native transition to modernity and 
modern settings with the culturally appropriate control beliefs. At present, no data have been collected to support the adaptive value of an expanded control beliefs system. However, findings concerning Druze gender differences in the endorsement of primary and secondary control beliefs may be indicative.

These findings showed that among 12th-graders, females scored significantly higher on primary control beliefs and males on secondary control beliefs. Note that upon completion of high school, only several months after the time of data collection, Druze males and females expected to move into different Israeli-Jewish settings. Males would start their compulsory military service, and many of the female respondents would attend college. Thus, as they plan to move into Israeli-Jewish settings, Druze adolescents adjust their control beliefs to fit more closely with the control beliefs prevailing in modern settings. Moreover, only as they get older do Druze adolescent females, like the American adolescents of the Band and Weisz study (1988), learn the culturally nonprevailing control beliefs. In the Druze adolescent case these are the primary control beliefs. However, the viability of this interpretation should be examined in a follow-up study testing two hypotheses: (1) as Druze females return to the more traditional lifestyle of their home villages their endorsement of primary and secondary control beliefs is readjusted to the prevailing cultural pattern (in itself a secondary control strategy); (2) as Druze males complete their military service and enter university their primary control beliefs become stronger and their secondary control beliefs weaker.

\section{CONCLUSION}

The two studies reported in this article were carried out independently. Because both have addressed adolescents' primary and secondary control beliefs in the cultural context of transition to modernity vs. modernity, we deemed it important to present them within the framework of one report and examine their results by addressing both their common and unique aspects.

This examination indicated that transition to modernity adolescents of different cultural settings endorsed secondary control beliefs more strongly than did their modern counterparts. Results concerning the primary control beliefs of transition to modernity adolescents relative to those of modern adolescents showed an inconsistent picture, and thus could not support the value-mediation hypothesis, and only partly supported the double transition hypothesis. Possible explanations for these results, grounded in respondents' characteristics and the nature of the instruments were presented in the Discussion, thus suggesting directions for future research.
In addition, this report brought to the fore two issues which bear on the conceptualisation and meaning of primary and secondary control: (1) the adaptive value of primary and secondary control beliefs; and (2) the explanatory value of the different control types. Both issues apply to individuals of different age groups and cultures, and to individuals experiencing other multiple transitions. Thus, it is suggested that the future agenda of the primary and secondary control beliefs research also include the study of their adaptive value and of the advisability of differentiation among different types of primary and secondary control. In the light of the results of the present studies these issues should be examined in relation to pertinent historical, developmental, and cultural contexts.

\section{REFERENCES}

Alsheich, S. (1978). Hazehut Hdruzit [Druze identity]. Unpublished master's thesis. Israel: Tel Aviv University (in Hebrew)

Band, E.B., \& Weisz, J.R. (1988). How to feel better when it feels bad: Children's perspectives on coping with everyday stress. Developmental Psychology, 24, 247-253. Beloff, H. (Ed.) (1986). Getting into life. London: Methuen.

Ben-Dor, G. (1979). The Druzes in Israel: A political study. Jerusalem: Magnes.

Brislin, R.W., Lonner, W.J., \& Thorndike, R.M. (1973). Cross-cultural research methods. New York: Wiley.

Essau, C. (1992). Primary-secondary control and coping: A cross-cultural comparison. Unpublished dissertation. Konstanz, Germany: University of Konstanz.

Essau, C., \& Trommsdorff, G. (1990). Kontrollwahrnehmung in individual- und gruppenorientierten Kulturen. In D. Frey (Ed.), Bericht über den 37. Kongre $\beta$ der Deutschen Gesellschaft für Psychologie in Kiel 1990. [Perception of control in individual and groupGesellschaft für Psychologie in Kiel 1990.
oriented cultures]. Göttingen: Hogrefe.

Essau, C., \& Trommsdorff, G. (1991). Primary and secondary control in different cultures. Unpublished paper. Konstanz, Germany: University of Konstanz.

Felner, R.D., Farber, S.S., \& Primavera, J. (1983). Transitions and stressful life events: A model for primary prevention. In R.D. Felner, L.A. Jason, J.N. Moritsugu, \& S.S. Farber (Eds), Preventive psychology: Theory, research and practice. New York: Pergamon, pp. 199-215.

Firru, K. (1984). Zehut Hadruzim: Haibet histori [Druze identity: The historical aspect]. In Soffer (Ed.), The Druzes in Israel. Haifa, Israel: The Jewish-Arab Center, University of Haifa (in Hebrew).

Frankl, V.E. (1963). Man's search for meaning: An introduction to logotherapy. New York: Washington Square Press.

Heckhausen, J., \& Schulz, R. (July 1991). Functional trade-offs in primary and secondary modes of control across the life course: conceptual issues and overview. Paper presented at the symposium on "Primary and secondary control across the life course: Developmenta and culture related differences". The 11th Biennial Meetings of the International Society for the Study of Behavioral Development. Minneapolis, MN.

Hui, C.H., \& Triandis, H.C. (1986). Individualism-collectivism: A study of cross-cultural researchers. Journal of Cross-Cultural Psychology, 17, 222-248. 
Marini, M.M. (1984). The order of events in the transition to adulthood. Sociology of Education, 57, 63-84

Markus, H.R., \& Kitayama, S. (1991). Culture and the self: Implications for cognition, emotion, and motivation. Psychological Review, 98(2), 224-253.

Paulhus, D. (1983). Sphere-specific measures of perceived control. Journal of Personality and Social Psychology, 44, 1253-1265.

Petersen, A.C. (1985). Pubertal development as the cause of disturbance: myths, realities, and unanswered questions. Genetic, Social, and General Psychology Monographs, 111, $205-232$.

Rothbaum, F., Weisz, J.R., \& Snyder, S.S. (1982). Changing the world and changing the self: A two-process model of perceived control. Journal of Personality and Social the self: A two-process

Rotter, J.B. (1966). Generalized expectancies for internal versus external control of reinforcement. Psychological Monographs, 80 (1, whole No. 609).

Schwartz, S.H. (1990). Individualism-collectivism: Critique and proposed refinements. Journal of Cross-Cultural Psychology, 21, 139-157.

Seginer, R., \& Halabi, H. (1991). Cross-cultural variations of adolescents' future orientation: The case of Israeli Druze versus Isareli Arab and Jewish males. Journal of CrossCultural Psychology, 22, 224-237.

Seligman, M.E.O. (1975). Helplessness: On depression, development, and death. San Francisco: Freeman.

Soffer, A. (Ed.) (1984). The Druzes in Israel. Haifa, Israel: The Jewish-Arab Center, University of Haifa (in Hebrew).

Triandis, H.C., Bontempo, R., Betancourt, H., Bond, M., Leung, K., Brenes, A., Goergas, J., Hui, C.H., Marin, G., Setiadi, B., Sinha, J.B.P., Verma, J., Spangenmberg, J., Touzard, H., \& deMontmollin, G. (1986). The measurement of etic aspects of individualism and collectivism across cultures. Australian Journal of Psychology, 38, 257-267. Trommsdorff, G. (1989). Socialization in Kulturvergleich. [Socialisation in cultural comparison]. Stuttgart: Ferdinant Enke.

Weisz, J.R. (1983). Can I control it? The pursuit of veridical answers across the life span. In P.B. Baltes \& O.G. Brim (Eds), Life-span development and behavior, Vol. 5. New York: Academic Press, pp. 233-300.

Weisz, J.R. (1986). Understanding the developing understanding of control. In M. Perlmutter (Ed.), The Minnesota Symposia on Child Psychology, Vol. 18. Hillsdale, NJ: Lawrence Erlbaum Associates Inc, pp. 219-278.

Weisz, J.R., Rothbaum, F. M., \& Blackburn, T.C. (1984). Standing out and standing in: The psychology of control in America and Japan. American Psychologist, 39, 955-969. 